\title{
A Dual-Focus Reflector Antenna for Spaceborne SAR Systems with Digital Beamforming
}

\author{
Sigurd Huber, Marwan Younis, Senior Member, IEEE, Gerhard Krieger, Senior Member, IEEE, \\ and Alberto Moreira, Fellow, IEEE
}

\begin{abstract}
Future spaceborne synthetic aperture radar (SAR) missions shall benefit from digital beamforming (DBF) techniques with the goal of generating high-resolution wide-swath imagery. The basic idea is to replace parts of the analog receiver hardware by digital components, increasing the flexibility of such systems. The limited transmit power rises the need of large antennas capable of electronically steering the antenna beam over a large angular domain. An innovative concept is the usage of large unfoldable mesh-reflector antennas in combination with digital feed arrays. An inherent problem of such systems is, that every feed element illuminates, after reflection from the main reflector, an essentially non-overlapping angular domain in the far field. In case of an element failure the radar system would be 'blind' in the specific direction. To circumvent costly redundant receiver hardware a new approach to increase the reliability of such systems has been studied. The approach is based on a so called defocused reflector which grants visibility of the complete angular domain under failure conditions. This article presents the electromagnetic considerations leading to the defocused reflector concept. The performance of the defocused reflector system is evaluated in comparison to a conventional parabolic reflector system, utilizing digital beamforming techniques.
\end{abstract}

Index Terms-DBF, reflector antennas, SAR, defocused

\section{INTRODUCTION}

$\mathbf{S}$ YNTHETIC aperture radar imaging is a vastly developing discipline, where information from distant objects can be retrieved contactless. Especially in the field of spaceborne earth observation missions the performance requirements in terms of revisit time or image resolution continuously increase. Since the radar antenna naturally represents the interface between the scattered electromagnetic energy and the radar signals after reception, a careful design of the antenna is one key step in the conception of an efficient SAR system. A requirement directly affecting the antenna design is the designated revisit time of the SAR satellite. For applications like earth system dynamics monitoring [1], a short revisit time is desired, which in turn rises the need of large swath widths. At the same time scientists are interested in a high information content of the SAR signal. This is traditionally achieved by increasing the signal bandwidth resulting in a higher resolution of the SAR images. Large swath widths and high azimuth resolutions are contradicting requirements for conventional SAR systems. This SAR inherent restriction can be overcome by digital beamforming techniques [2], [3].

Manuscript received November 18, 2011. S. Huber, M. Younis, G. Krieger and A. Moreira are with the Microwaves and Radar Institute, German Aerospace Center (DLR), Oberpfaffenhofen, Germany, e-mail: sigurd.huber@dlr.de.
Of increasing interest for SAR applications become large reflector antennas in combination with feed arrays [3]-[5]. These systems are already a mature technique for communication satellites, where commercial deployable reflectors with diameters of more than $22 \mathrm{~m}$ and surface accuracies up to Kaband are realizable [6]. Reflector antennas typically generate a shaped beam due to the mechanical molding of the reflector dish. That means, for any given feed position, only a certain solid angle can be illuminated. Therefore a set of multiple feed elements is required in order to cover the complete region of interest. This technology is considered as baseline for Tandem-L [7], a mission proposal for an interferometric Lband radar instrument. In [8] a reflector based Ka-Band system for high resolution Earth observation applications has been investigated. Evidently such systems are severely handicapped in case of a feed element failure. A dropped out element would cause a 'blind' spot in the antenna footprint. Therefore, state-of-the-art systems are planed with redundant receiver hardware. Clearly this option poses a considerable increase of the costs for satellite SAR systems. Moreover, the redundant electronics, as for example additionally required switches, will produce losses.

The most widely established spaceborne SAR antenna is the planar phased array antenna. This kind of antenna is very robust against a single or even multiple element failures in terms of beam shape stability. The drawback is clearly that the antenna size required to realize a high-resolution wide-swath SAR system can render a SAR mission infeasible concerning the costs. Consequently, an antenna design is needed, which combines the robustness against element failure of a planar array antenna with the high gain of a large unfoldable lowweight reflector antenna. This concept involves a defocusing of the individual element beams by utilizing a new reflector surface.

This article is organized as follows. Section II explains the system operation concept of reflector based SAR systems. Section III introduces the concept of defocused reflector antennas. Section IV demonstrates the performance, considering the aspect of feed element failure. After a discussion in section $\mathrm{V}$, the paper is concluded in section VI.

\section{Spaceborne SAR with ReFlector Antennas}

The operation principle of reflector-based SAR systems with linear digital feed arrays is sketched in Fig. 1, with the flight direction orthogonal to the paper plane. The radar pulse, commonly a chirp waveform, is transmitted with all 


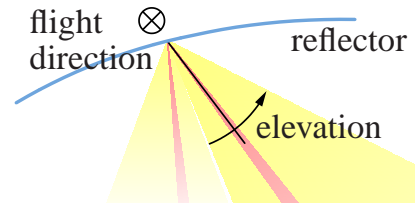

DBF

feed array

swath

Fig. 1. Reflector SAR system operation with transmit pattern (yellow) and receive beam (red).

feed elements active simultaneously, indicated by the yellow pattern. After deflection by the reflector the pulse impinges the ground and moves from the near to the far end of the swath. The signal returning from the ground is again scattered by the reflector and successively collected by different parts of the feed array. This is illustrated by the red receive beam, scanning the swath in elevation direction. This mode of operation is known as SCORE first suggested by [9] and further developed by [10] and [11]. Note that in Fig. 1 the receive beam on the feed array moves exactly the opposite direction as the receive beam on ground. That means the most right feed element 'sees' the near end of the swath.

It becomes clear that in case of an element failure a certain part of the elevation scan angle domain becomes inaccessible, excluding the option of mechanical steering. A possible solution is to defocus the reflector such that adjacent feed elements illuminate the same region on ground. A defocusing always involves a broadening of the beams and a loss of gain, or signal-to-noise-ratio $(S N R)$, respectively. This effect is usually not desirable. For example communication satellites are optimized to illuminate a continuum of directions, that is for example a whole continent, with a high gain pattern simultaneously. However, in a SAR application, the signal changes its direction of arrival over time, as it travels over ground. That means for every time instance a new beam is formed digitally onboard the satellite.

Since the receive beam is scanned in elevation, it is mandatory that the reflector is defocused only in this direction, in order to avoid gain loss. All signal energy radiated in the orthogonal direction, namely azimuth or the flight direction, respectively, is lost for the linear feed array.

\section{Defocused Reflector Antennas}

The concept of defocusing a reflector antenna is not new. Publications like [12]-[14] achieve a defocusing by moving the feed out of the focal point and leave the shape of the reflector antenna unaltered. The advantage is that conventional reflectors with paraboloidal, cylindrical or corner surfaces can be utilized. If however a defocusing is desired in only one dimension, elevation in this example, a simple displacement of the feed array would not be satisfying, since the pattern tends to symmetrically defocus in all dimensions. For example a paraboloidal reflector with an axially shifted feed will,

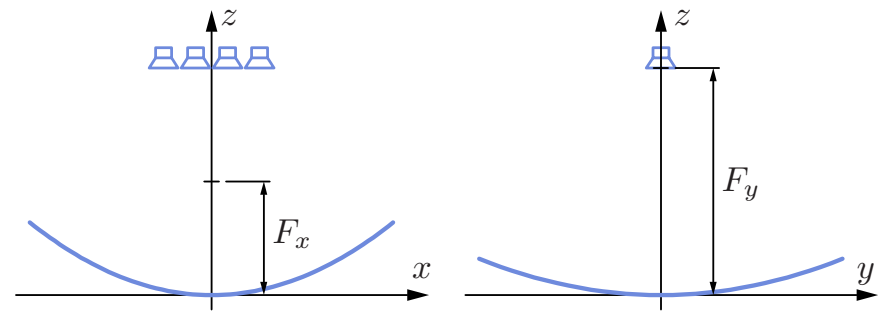

Fig. 2. Cut drawings of a parabolic reflector with two foci. The linear feed array, indicated by the antenna symbols, is placed at the upper focal point at $\left[0,0, F_{y}\right]$.
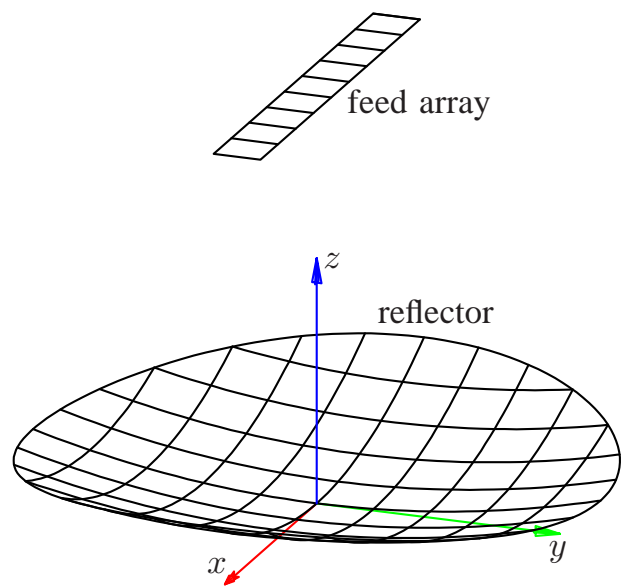

Fig. 3. Perspective presentation of the dual-focus reflector antenna with feed array in the upper focal point.

compared to the focused case, generate a broader rotational symmetric Bessel-like beam.

The favoured method to defocus a reflector antenna is to shape the reflector surface itself. The starting point is a parabolic reflector. Such a shape is generated by rotating a parabola for example around the $z$-axis, as indicated in Fig. 2. Characteristic for such shapes is, that a plane wave incident anti-parallel to the $z$-axis is focused in the so called focal point. A simple method to defocus a parabolic reflector is to construct a reflector with two focal points. Such a geometry can be described in Cartesian coordinates as

$$
z=\frac{x^{2}}{4 F_{x}}+\frac{y^{2}}{4 F_{y}}
$$

where $F_{x}$ and $F_{y}$ denote the focal lengths in the $x$ - and $y$-dimension, respectively, as given in the cut drawings in Fig. 2. Fig. 3 shows a perspective view of the dual-focus reflector antenna with circular projected aperture. The linear feed array is placed in the upper focal plane parallel to the $x$ axis in a centered position. Here the feed elements are simply directed in negative $z$-direction. In a real SAR system, the outer feed elements could be tilted slightly, in order to provide a more homogeneous illumination of the reflector. Also bent feed array structures might be of interest. The defocusing effect has been analyzed by simulation of the two-dimensional electromagnetic field distribution in the focal plane $z=F_{y}$. Fig. 4 shows the $z$-component of the power flux density or 


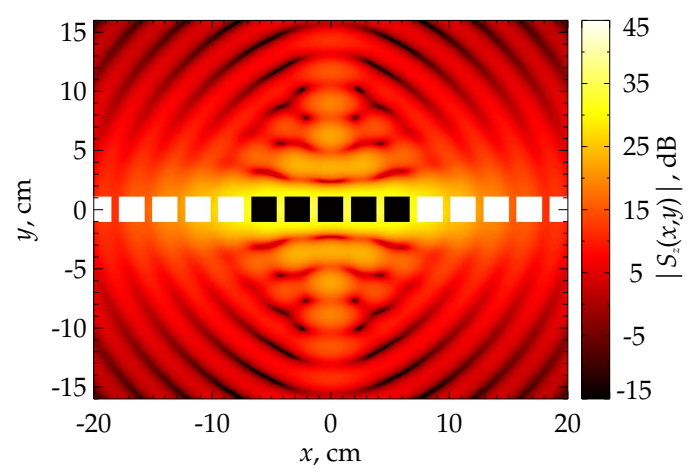

Fig. 4. Power density distribution in the upper focal plane for a defocused reflector with two foci. The squares represent feed array elements, of which the black ones denote elements covered by the field.

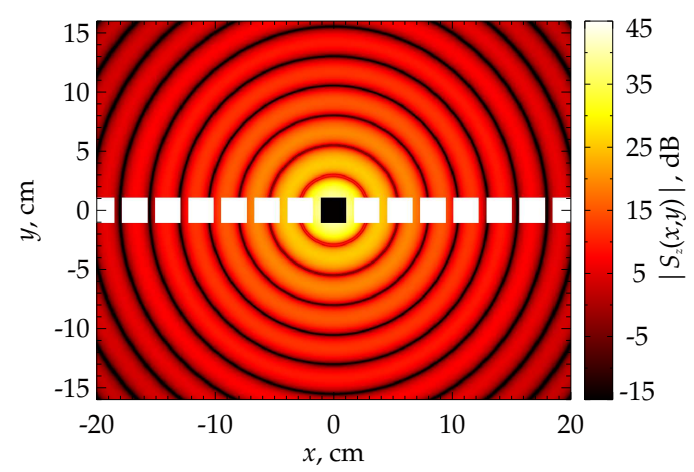

Fig. 5. Power density distribution for a conventional single-focus parabolic reflector in the focal plane. The squares represent feed array elements, of which the black one denotes the element covered by the field.

Poynting vector

$$
\mathbf{S}=\mathbf{E} \times \mathbf{H}
$$

as function of the Cartesian coordinates $x$ and $y$. Note that the $x$-direction can be associated with elevation and the $y$ direction with azimuth. This power density distribution is the result of a plane wave arriving anti-parallel to the $z$-direction and being scattered by the dual-focus reflector. Clearly one can observe, that the power density is concentrated in azimuth direction and distributed over a larger domain in the elevation direction, as desired by design. The squares shall represent the elements of the linear feed array. Here, black squares denote feed elements which are covered by the electromagnetic field above a given threshold. Consequently those feed elements 'see' the afore mentioned plane wave and serve as redundant elements in case of a drop out. For comparison, the power density distribution for a conventional single-focus reflector is depicted in Fig. 5. The power density is concentrated around the focal point as expected. In case of a failure of the center element, the neighboring elements are not able to catch enough field energy to compensate the gain loss.

As expected from parabolic reflector antenna theory, the phase pattern $\arg \left\{S_{z}\right\}$ in the region around the focal point is approximately constant for the above plane wave scenario. In
TABLE I

REFLECTOR ANTENNA PARAMETERS.

\begin{tabular}{l|c} 
parameter & value \\
\hline frequency & $9.65 \mathrm{GHz}$ \\
reflector diameter & $7 \mathrm{~m}$ \\
focal length (single-focus) & $5 \mathrm{~m}$ \\
focal lengths (dual-focus) & $F_{x}=4.95 \mathrm{~m}, F_{y}=5.05 \mathrm{~m}$ \\
number of feed elements & 35 \\
feed element spacing (single-focus) & $0.9 \lambda$ \\
feed element spacing (dual-focus) & $(0.8 \cdots 0.9) \lambda$
\end{tabular}

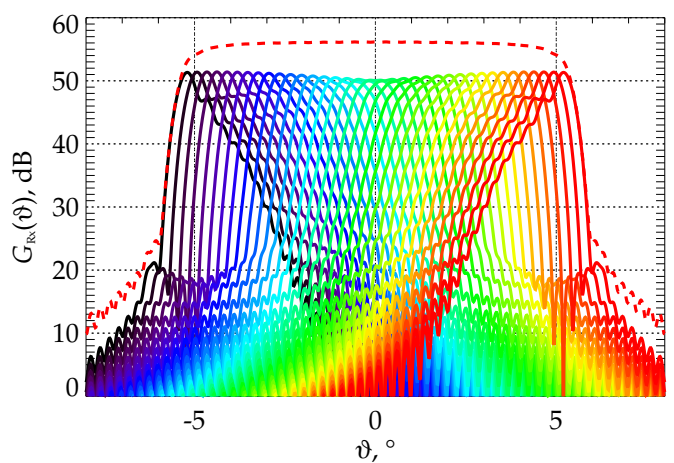

Fig. 6. Co-polar gain patterns for the dual-focus reflector with 35 feed elements. The solid lines represent the individual patterns while the dashed line shows the pattern after digital beamforming on receive.

contrast in the dual-focus case phase variations in the order of $500^{\circ}$ can be observed.

\section{PERFORMANCE}

The more common way to examine an antenna's performance is to look at the gain patterns. Therefore the coand cross-polar components of the electric far field for a reflector antenna with the specifications given in Tab. I have been simulated, using the EM simulation software TICRA GRASP [15]. Here each feed element pattern is simulated by a linearly polarized Gaussian beam. For reciprocity reasons from the power density distribution shown in Fig. 4 it can be expected that the individual antenna patterns will also broaden and suffer a gain loss. This can be observed in the elevation cut plots in Fig. 6. Each gain pattern corresponds to a feed element, or channel. The most right pattern in Fig. 6 belongs to the most left feed element in Fig. 4 (not visible in this plot). Again, for comparison, the analogous plot for a conventional single-focus reflector antenna is shown in Fig. 7. Here, the sharp angular separation between the individual channel patterns can be recognized.

Clearly, the defocused reflector antenna unfolds its full potential when digital beamforming techniques are applied. The innovative hardware structure of such DBF SAR systems foresees a digitization of the individual channel signals almost immediately after the receiver. These signals are then processed either onboard the satellite by means of field programmable gate arrays (FPGA) or at the ground station. A DBF technique known as minimum variance distortionless 


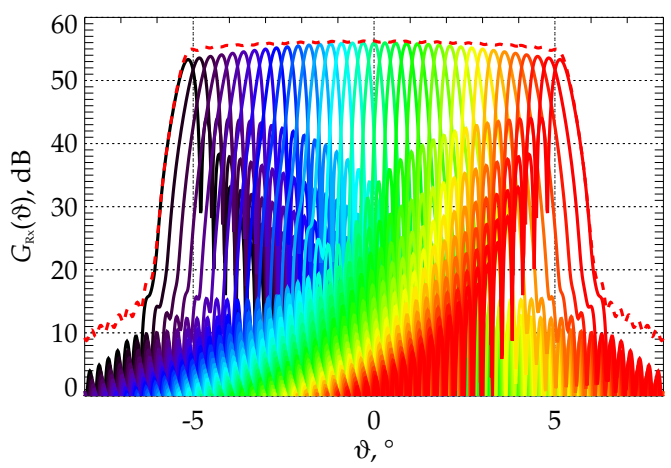

Fig. 7. Co-polar gain patterns for the single-focus reflector with 35 feed elements. The solid lines represent the individual patterns while the dashed line shows the pattern after digital beamforming on receive.

response (MVDR) [16] beamforming optimizes the signal-tonoise-ratio of a beamformer output

$$
u_{\mathrm{DBF}}(t)=\mathbf{w}^{\top}(\vartheta)[\mathbf{a}(\vartheta) s(t)+\mathbf{n}(t)]
$$

where $\mathbf{w}$ is a complex weight vector, $\mathbf{a}$ is the array manifold, $s$ is the signal of interest and $\mathbf{n}$ represents the thermal receiver noise in each channel. The MVDR weights are given as

$$
\mathbf{w}^{*}(\vartheta)=\frac{\mathbf{R}_{\mathbf{n}}^{-1} \mathbf{a}(\vartheta)}{\mathbf{a}^{\mathrm{H}}(\vartheta) \mathbf{R}_{\mathbf{n}}^{-1} \mathbf{a}(\vartheta)},
$$

where $(.)^{\mathrm{H}}$ means conjugate transpose (Hermitian) and $\mathbf{R}_{\mathbf{n}}$ is the noise channel covariance matrix. Generally, for any choice of the weight vector, the precise knowledge of the embedded complex array patterns is relevant. That means, effects from multi-path propagation or reflector surface imperfections, which potentially deteriorate the single channel characteristic, will not impact severely the beam shape after applying the complex weights. Insofar digital beamforming allows to tolerate even unknown error sources to a certain degree, as long as the array manifold is known accurately enough. In practice this knowledge is obtained via a calibration campaign over rain forest. Equation (3) is written with mixed variables $t$ for time and $\vartheta$ for the elevation angle. This can be done since there is a functional relationship, given by the imaging geometry, between those two variables. For every time instance, or elevation angle, a new set of complex weights is computed. The dashed red curves in Figs. 6 and 7 represent the patterns after digital beamforming on receive. Here for the purpose of illustration the noise has been neglected and the signal has been set to one. For the array manifold a simply the co-polar electric field is inserted, yielding

$$
\mathbf{a}(\vartheta)=\left[\begin{array}{llll}
E_{1}(\vartheta) & E_{2}(\vartheta) & \ldots & E_{N}(\vartheta)
\end{array}\right]_{\mathrm{co}-\mathrm{pol}}^{\top} .
$$

In our case $N$ is 35 . Substituting equation (4) into (3) the resulting pattern, as plotted with dashed red lines in Figs. 6 and 7 , is then

$$
G(\vartheta)=\mathbf{a}^{\mathrm{H}}(\vartheta) \mathbf{a}(\vartheta)
$$

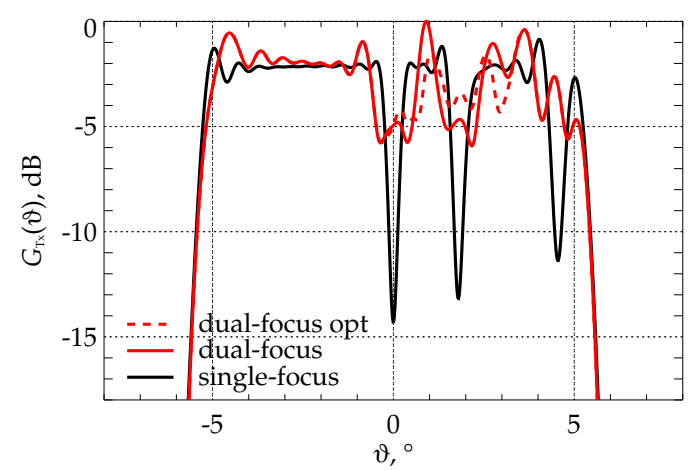

Fig. 8. The solid lines represent the normalized gain patterns on transmit for the single- and the dual-focus reflector. Indicated by the dashed line is a heuristically optimized pattern, using phase-only synthesis methods.

\section{A. Performance under Failure Conditions}

The initial question was how such a defocused reflector SAR system performs when a feed element fails. Basically two failure scenarios can be distinguished, a disruption in the transmit paths, for example caused by a loss of high power amplifiers (HPA), and failures in the receive chain. Fig. 8 shows the normalized transmit gain pattern when elements 18, 24 and 33 , counting from right to left, fail. Such a large number of lost elements is an unrealistic scenario and serves here only for illustration purposes. For instance, the German SAR satellite TerraSAR-X uses phased array antenna technology with 384 transmit/receive modules. Since the launch of the satellite five years ago, all T/R modules are working properly. The singlefocus system sustains a $12 \mathrm{~dB}$ gain loss at the center of the pattern, decreasing towards the border of the pattern. The defocused reflector is able to keep the gain loss on a constant level of roughly $3 \mathrm{~dB}$. Indicated by the dashed red line is the result of a phase-only pattern synthesis, taking into account the four elements 22, 23, 25 and 26 around the failed element 24. This attempt shows that there might be the potential to further reduce the effects from a lost element on transmit for defocused reflector systems by a proper weight selection.

On receive, the situation is different. Here at every time instance, or at every elevation angle, respectively, a new beam is formed. However, even with the MVDR beamformer the single-focus reflector is prone to large gain losses in the order of $12 \mathrm{~dB}$ at the pattern center, as indicated in Fig. 9. The potential of the defocused reflector in combination with digital beamforming techniques becomes evident, when looking at the solid red curve in Fig. 9. With MVDR beamforming the gain loss can be significantly reduced. As reference the pattern is plotted, when no special DBF techniques are applied (dashed red curve and denoted with unity). In this case only the five elements receiving the highest power (compare to Fig. 4) are activated on receive.

\section{DiscusSION}

As stated in the beginning, a major cost driver for a reflector based SAR system is the feed array, including feed antennas and electronics. In order to minimize the number of feed 


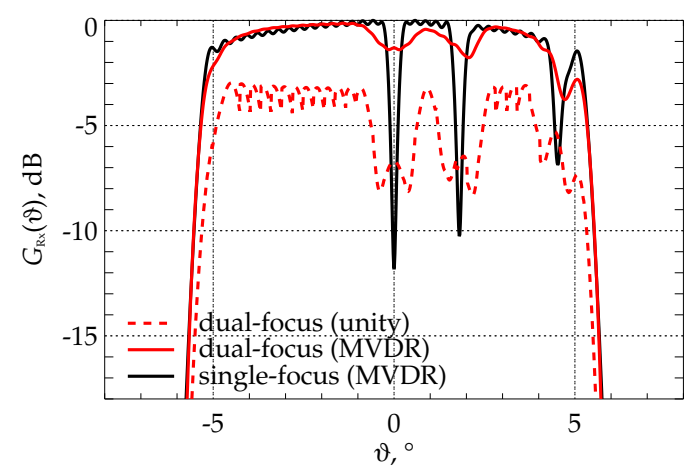

Fig. 9. Normalized gain pattern after digital beamforming, with elements 18 , 24 and 33 dropped out. The dashed line shows the receive pattern without DBF techniques, when only five elements are turned on at a time instance.

elements a relatively large feed element spacing of 0.8 to $0.9 \lambda$ (see Tab. I) has been chosen. As can be observed in Fig. 9 this causes a basically unwanted ripple on the gain pattern after digital beamforming for the conventional single-focus reflector system. With the dual-focus system the ripple vanishes and a smooth gain pattern over the entire swath can be realized. Despite the defocusing it shows that reflector SAR systems in general suffer especially, if an element at the border of the array fails. The consequence is a reduced swath-width, which can be illuminated adequately. A possible strategy is to add two or three feed elements on both sides, which serve as redundant elements. This is still a more cost efficient solution than doubling the whole number of transmit-receive modules. A more general approach could be to optimize the reflector surface together with the feed array. This might involve feed arrays with new geometrical shapes. As demonstrated in the previous section, defocused reflector SAR systems unfold their full potential only in conjunction with DBF techniques.

Of special importance for polarimetric SAR applications is the polarization purity. Reflector antennas can introduce crosspolarized signal power, contaminating the co-polar signal, in two ways, first by the reflector itself and second by the feed antenna. Here we concentrate on the effects induced by the reflector assuming ideal feed antennas. Field simulations of the cross-polar patterns show in case of the single-focus reflector, that the gain maximum varies between $-13.9 \mathrm{~dB}$ for the center element and $13.0 \mathrm{~dB}$ at the feed array edges, while in the dualfocus case the gain maximum for the same feed elements varies between $-10.8 \mathrm{~dB}$ and $10.4 \mathrm{~dB}$, respectively. Although the cross-polar levels for the center feed element of the dualfocus reflector rises, this analysis shows that the defocusing seems to balance the peak cross-polar levels and therefore does not effect polarimetric operation negatively.

In real SAR systems essentially two types of feed antennas, depending on the frequency band, are of interest. For example in L-band or P-band systems commonly use patch antennas, while in X-band also horn antennas are an alternative. Due to the dense packing of the feed antennas, effects from coupling become more pronounced compared to direct radiating arrays with large inter-element spacings. However, with the same reasoning as in section IV, the performance after digital beamforming will be impacted only marginally, assuming a precise knowledge of the complex patterns.

\section{CONCLUSION}

Future synthetic aperture radar systems based upon reflector antennas with digital feed arrays are in need of redundant receiver hardware, because a feed element failure will leave a blind spot in the swath to be imaged. This article introduced the innovative concept of a defocused reflector. Instead of moving the feed array out of the focal plane, the defocusing has been achieved by shaping the reflector surface itself. This gives the unique advantage of one-dimensional defocused antenna patterns. A surface with the potential to generate such patterns was shown to be a double-parabolic reflector with two focal points, where the feed array is centered in one of the two foci. In case of a feed element failure, the neighboring element patterns ensure a sufficient visibility of the otherwise blind spot. The inevitable gain loss for the individual element patterns is compensated by digital beamforming techniques, where the individual channel signals are combined to a single high gain signal. Such defocused reflector concepts have the potential to significantly reduce the costs for spaceborne SAR systems.

\section{REFERENCES}

[1] G. Krieger, I. Hajnsek, K. Papathanassiou, M. Eineder, M. Younis, F. D. Zan, S. Huber, P. Lopez-Dekker, P. Prats, M. Werner, Y. Shen, A. Freeman, P. Rosen, S. Hensley, B. Johnson, L. Villeux, B. Grafmüller, R. Werninghaus, R. Bamler, and A. Moreira, "Tandem-L: An Innovative Interferometric and Polarimetric SAR Mission to Monitor Earth System Dynamics with High Resolution," in IEEE International Geoscience and Remote Sensing Symposium (IGARSS), Jul 2010, pp. 253-256.

[2] S. Huber, M. Younis, A. Patyuchenko, and G. Krieger, "Digital Beam Forming Techniques for Spaceborne Reflector SAR Systems," in 8th European Conference on Synthetic Aperture Radar (EUSAR), Jun 2010, pp. 962-965.

[3] G. Krieger, N. Gebert, M. Younis, and A. Moreira, "Advanced Synthetic Aperture Radar Based on Digital Beamforming and Waveform Diversity," in Radar Conference, 2008. RADAR '08. IEEE, May 2008, pp. 767-772.

[4] M. Younis, S. Huber, A. Patyuchenko, F. Bordoni, and G. Krieger, "Performance Comparison of Reflector- and Planar-Antenna based Digital Beam-Forming SAR," International Journal of Antennas and Propagation, vol. 2009, pp. 1-14, Jun 2009.

[5] A. Freeman, G. Krieger, P. Rosen, M. Younis, W. Johnson, S. Huber, R. Jordan, and A. Moreira, "SweepSAR: Beam-forming on Receive using a Reflector-Phased Array Feed Combination for Spaceborne SAR," in IEEE Radar Conference (RadarCon), May 2009.

[6] "Commercial Deployable (Unfurlable) Antenna Reflectors," http://www.govcomm.harris.com/solutions/products/isr/deployantennas-reflectors.asp, Harris Corporation, Aug 2012.

[7] A. Moreira, G. Krieger, M. Younis, I. Hajnsek, K. Papathanassiou, M. Eineder, and F. D. Zan, "Tandem-L: A Mission Proposal for Monitoring Dynamic Earth Processes," in IEEE International Geoscience and Remote Sensing Symposium (IGARSS), Jul 2011, pp. 1385-1388.

[8] M. Ludwig, S. D'Addio, and P. Saameno-Perez, "Ka-Band SAR for Spaceborne Applications based on Scan-on-Receive Techniques," in European Conference on Synthetic Aperture Radar (EUSAR), Jun 2008.

[9] J. H. Blythe, "Radar systems," U.S. Patent 4253 098, Feb, 1981.

[10] J. T. Kare, "Moving receive beam method and apparatus for synthetic aperture radar," U.S. Patent 6175326 , Jan, 2001.

[11] M. Suess and W. Wiesbeck, "Side-looking synthetic aperture radar system," European Patent EP 1241 487, Sep, 2002.

[12] D. Cheng and S. Moseley, "On-Axis Defocus Characteristics of the Paraboloidal Reflector," IRE Transactions on Antennas and Propagation, pp. 214-216, Oct 1955. 
[13] R. Yang, "Illuminating curved passive reflector with defocused parabolic antenna," in WESCON/58 Conference Record, vol. 2, Aug 1958, pp. 260-265.

[14] A. Olver and J. Syed, "Variable beamwidth reflector antenna by feed defocusing," IEE Proceedings - Microwaves, Antennas and Propagation, vol. 142, no. 5, pp. 394-398, Oct 1995.

[15] "General reflector and antenna farm analysis software," http://www.ticra.com, TICRA, Aug 2011.

[16] H. L. V. Trees, Optimum Array Processing. John Wiley \& Sons, Inc., 2002.

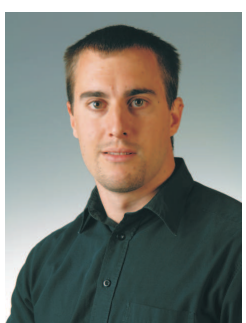

Sigurd Huber received the Dipl.-Ing. (M.S.) degree in electrical and communication engineering from the Technical University of Munich (TUM), Germany, in 2005. Since 2005 he works as a scientist at the Microwaves and Radar Institute of the German Aerospace Center (DLR), Oberpfaffenhofen, Germany.

$\mathrm{He}$ is involved in national and international projects in the field of synthetic aperture radar, encompassing cooperations with industry partners (EADS Astrium), as well as research organizations (ESA, NASA/JPL). In the frame of his Ph.D. thesis he is concerned with the conception of future SAR systems, utilizing advanced multichannel hardware architectures, based on array fed reflector antennas with digital beamforming. He contributed as author and co-author in more than 30 conference papers and four peer reviewed journal articles. His research interests include digital signal processing, electromagnetic field theory and inverse problems.

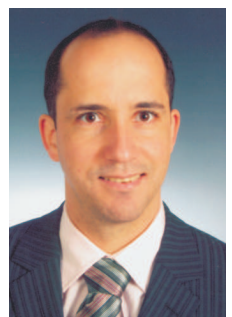

Marwan Younis (S1995, M2005, SM2008) was born in Las Cruces, USA., in 1970. He received his B.Sc in electrical engineering from the University of Baghdad, Iraq in 1992 and the Dipl.-Ing. (M.Sc.) and Dr-Ing. (Ph.D.) degree in electrical engineering from the Universität Karlsruhe (TH), Germany, in 1997 and 2004, respectively.

From 1998 to 2004, he was a research scientist with the Institut für Höchstfrequenztechnik und Elektronik, Universität Karlsruhe (TH). Since 2005 he has been with the Microwaves and Radar Institute of the German Aerospace Center (DLR), Oberpfaffenhofen, Germany. He is the author and co-author of over 70 conference papers and more than 15 reviewed publications. His research fields include synthetic aperture radar (SAR) systems, SAR performance, digital beamforming for radar, synchronization of bistatic SAR, forward looking radar, and antennas.

Dr. Younis is an active member of the IEEE and the German Association for Location and Navigation (DGON). He is the active microwave working group leader within the IEEE instruments and future technologies technical committee. He is a lecturer with the Universität Karlsruhe (TH) and the CarlCranz-Gesellschaft. He received the Hermann-Billing award for his Ph.D. thesis in 2005.

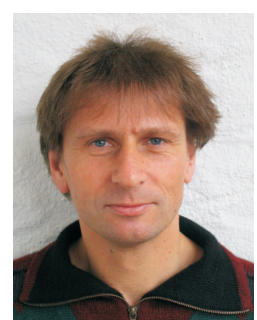

Gerhard Krieger received the Dipl.-Ing. (M.S.) and Dr.-Ing. (Ph.D.) degrees (with honors) in electrical and communication engineering from the Technical University of Munich, Germany, in 1992 and 1999, respectively.

From 1992 to 1999, he was with the LudwigMaximilians University, Munich, where he conducted multidisciplinary research on neuronal modeling and nonlinear information processing in biological and technical vision systems. In 1999, he joined the Microwaves and Radar Institute (HR) of the German Aerospace Center (DLR), Oberpfaffenhofen, Germany, where he developed signal and image processing algorithms for a novel forward looking radar system employing digital beamforming on receive. From 2001 to 2007 he led the New SAR Missions Group which pioneered the development of advanced bistatic and multistatic radar systems as exemplified by the forthcoming TanDEM-X mission as well as innovative multi-channel SAR techniques and algorithms for high-resolution wide-swath SAR imaging. Since 2008, he has been Head of the new Radar Concepts Department of the Microwaves and Radar Institute, DLR, Oberpfaffenhofen, Germany.

Dr. Krieger received several national and international awards, including the W.R.G. Baker Prize Paper Award from the IEEE board of directors and the IEEE Transactions Prize Paper Award of the Geoscience and Remote Sensing Society. He is author of about 50 peer reviewed journal papers, 4 invited book chapters, more than 200 conference papers, and 5 patents. His current research interests focus on the development of multi-channel radar techniques and algorithms for innovative MIMO SAR systems, the demonstration of novel interferometric and tomographic Earth observation applications, and the conceptual design of advanced bi- and multistatic radar missions.

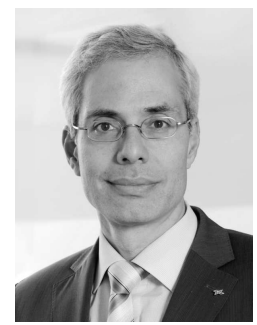

Alberto Moreira (S'96-F'04) received the B.S.E.E. and M.S.E.E. degrees from the Aeronautical Technological Institute (ITA), São José dos Campos, in 1984 and 1986, respectively, and the Eng. Dr. degree (with honors) from the Technical University of Munich, Germany, in 1993. From 1996 to 2001, he was the Chief Scientist and Engineer with the SAR Technology Department, German Aerospace Center (DLR), Oberpfaffenhofen, Germany. Under his leadership, the DLR airborne SAR system has been upgraded to operate in innovative imaging modes like polarimetric SAR interferometry and SAR tomography. Since 2001, he has been the Director of the Microwaves and Radar Institute at DLR. The Institute contributes to several scientific programs and space projects for actual and future airborne and spaceborne SAR missions like TerraSAR-X, TanDEM-X and Sentinel-1. The mission TanDEM-X, led by his Institute, has successfully started the operational phase in December 2010. Prof. Moreira is the initiator and Principal Investigator for this mission. Since 2003, he is also a Full Professor with the Karlsruhe Institute of Technology, Germany, in the field of microwave remote sensing. He has more than 300 publications in international conferences and journals and is the holder of 15 patents in the radar and antenna field. His professional interests and research areas encompass radar end-to-end system design and analysis, innovative microwave techniques and system concepts, signal processing, and remote sensing applications. He is a member of the IEEE Geoscience and Remote Sensing Society (GRSS) Administrative Committee (1999-2001, 2004-2013, 2010 as President, 2011-2013 as Past-President), was the Founder and Chair of the GRSS German Chapter (2003-2008) and Associate Editor for the IEEE GRS Letters (2003-2007) and for the IEEE TGRS (2005-2011). He and his colleagues received the GRS-S Transactions Prize Paper Awards in 1997, 2001, and 2007 and the IEEE W.R.G. Baker Award in 2012. He is also the recipient of the DLR Science Award (1995), IEEE Nathanson Award for the Young Radar Engineer of the Year (1999) and the IEEE Kiyo Tomiyasu Field Award (2007). From 2003 until 2008, he served as a member of the Board of Directors of the Information Technology Society of the German Association for Electrical, Electronic and Information Technologies and as chair of the Scientific and Technical Council of DLR (2009-2011). He has contributed to the successful series of the European SAR conferences (EUSAR) since 1996 as a member of the Technical Program Committee, Technical Chairman (2000), Awards Chairman (2002-2004), General Chairman (2006), and CoChairman (2008) and is severing as General Co-Chair for IGARSS' 12 in Munich. 